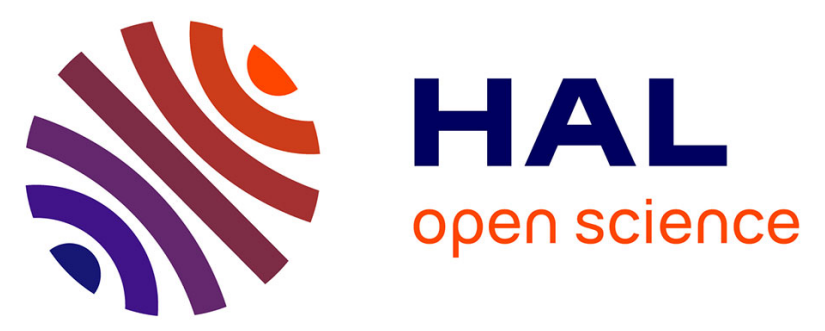

\title{
La reconstruction de chaînes d'impacts au service de l'évaluation de la résilience des territoires et de la réduction des risques météo-marins : le cas des atolls des Tuamotu, Polynésie française
}

\author{
Virginie Duvat, Alexandre K. Magnan, Rémy Canavesio
}

\section{To cite this version:}

Virginie Duvat, Alexandre K. Magnan, Rémy Canavesio. La reconstruction de chaînes d'impacts au service de l'évaluation de la résilience des territoires et de la réduction des risques météo-marins : le cas des atolls des Tuamotu, Polynésie française. Colloque Risques et résilience des territoires: apports de la notion de résilience à la gestion des risques, SHF, Nov 2017, Marne La Vallée, France. hal-01634349

\author{
HAL Id: hal-01634349 \\ https://hal.science/hal-01634349
}

Submitted on 14 Nov 2017

HAL is a multi-disciplinary open access archive for the deposit and dissemination of scientific research documents, whether they are published or not. The documents may come from teaching and research institutions in France or abroad, or from public or private research centers.
L'archive ouverte pluridisciplinaire HAL, est destinée au dépôt et à la diffusion de documents scientifiques de niveau recherche, publiés ou non, émanant des établissements d'enseignement et de recherche français ou étrangers, des laboratoires publics ou privés. 


\title{
La reconstruction de chaînes d'impacts au service de l'évaluation de la résilience des territoires et de la réduction des risques météo-marins : le cas des atolls des Tuamotu, Polynésie française
}

\author{
Virginie K.E. DUVAT ${ }^{1}$, Alexandre K. MAGNAN ${ }^{2,1}$, Rémy CANAVESIO ${ }^{3}$ \\ ${ }^{1}$ UMR LIENSs 7266, Université de la Rochelle-CNRS, Bâtiment ILE, 2 rue Olympe de Gouges, 17000 La Rochelle - \\ virginie.duvat@univ-lr.fr \\ 2 Iddri, 27 rue Saint-Guillaume, 75007 Paris - alexandre.magnan@iddri.org \\ ${ }^{3}$ CNRS-LETG 6554 Caen Géophen, Université de Caen Normandie - remycanavesio@hotmail.fr
}

\begin{abstract}
Cet article démontre l'intérêt d'une approche de la résilience des territoires par la reconstruction de "chaînes d'impacts" d'événements passés. Proposé au Ministère de l'Ecologie comme composante du référentiel national d'adaptation au changement climatique [ONERC, 2012], ce concept a fait l'objet d'applications à des cyclones tropicaux ayant touché les atolls des Tuamotu (Polynésie française) dans le cadre du projet RDT Réomers (2013-2016). Les résultats révèlent: (1) L'ampleur et la variabilité des impacts des cyclones, riches d'enseignements pour renforcer la résilience. (2) Le rôle crucial des retours d'expérience passés dans la réduction de la vulnérabilité, à travers le renforcement de la prévention et la sécurisation des ressources vitales. (3) Les barrières à la réduction des risques et à l'adaptation (éclatement territorial, conflits d'acteurs et manque de moyens financiers mobilisables, notamment). (4) Les mécanismes complexes, tant physiques (exhaussement des îles) que socio-économiques (amélioration de la productivité agricole et des conditions de vie), par lesquels les cyclones passés ont contribué à construire la résilience, et qui impliquent de penser des politiques d'adaptation spécifiques et intégrées. (5) L'émergence de nouvelles formes de vulnérabilité, dues à une augmentation de la dépendance des atolls vis-à-vis de Tahiti (importations alimentaires et dépendance associée aux emplois rémunérés, problèmes de santé publique). La méthode utilisée est (i) applicable à tout territoire et à toute perturbation; (ii) permet d'identifier des leviers pour réduire les risques actuels; (iii) répond aux enjeux de l'adaptation au changement climatique.
\end{abstract}

Chaînes d'impacts, Résilience, Adaptation, Atolls, Polynésie française

\section{Reconstructing " chains of impacts 》 to assess a territory's resilience and support coastal risk reduction}

This paper defends the interest of addressing a territory's resilience based on the reconstruction of "chains of impacts" of past catastrophic events. The concept of "chain of impacts", which had been proposed to the French Ministry of Ecology to be included in the national strategy on adaptation to climate change [ONERC, 2012], has been applied to tropical cyclones that hit the Tuamotu atolls (French Polynesia) in the framework of the RDT research project Réomers (2013-2016). The results highlight: (1) The intensity and high variability of tropical cyclones impacts, from which key lessons can be learnt to strengthen resilience. (2) The crucial role of the lessons learnt from past events in the reduction of these atolls'vulnerability based on prevention enhancement and vital resources securing, in particular. (3) The barriers to risk reduction and adaptation to climate change (archipelagic structure of the Tuamotu, stakeholders' conflicts and lack of financial means). (4) The complex and intertwined processes, both physical (sediment supply allowing island upward growth) and socio-economic (increase in agriculture productivity and improvement of living conditions), through which past cyclones have contributed to the strengthening of atoll resilience, which emphasize the need for atoll-specific and integrated adaptation to climate change strategies. (5) The new forms of vulnerability that were generated by the processes driving resilience, which increased atolls dependency towards Tahiti concerning food supply (increase in food imports and relating dependency on cash work, and induced health problems). Importantly, the method used to reconstruct chains of impacts: (i) is applicable to any type of territory and event; (ii) allows identify levers to reduce current risks; (iii) addresses the adaptation to climate change challenges.

Chains of impacts, Resilience, Adaptation, Atolls, French Polynesia

\section{INTRODUCTION}

La résilience peut être définie comme étant la capacité d'un système complexe, ici le territoire, à faire face à des perturbations sans perdre son identité et sans changer de nature [Walker et al., 2004]. Cette définition 
implique une approche globale (prise en compte de l'ensemble des composantes du système), systémique (focalisation sur l'analyse des interactions qui contrôlent les réponses du système aux perturbations), dynamique (centrage sur l'évolution du système à travers le temps) et territorialisée (un système étant défini par ses caractéristiques intrinsèques). Sur ces bases, une approche par la résilience consiste à évaluer l'évolution de la capacité de réponse d'un système, autrement dit à explorer les modalités et processus de retour à l'équilibre, et les mécanismes d'ajustement qui les contrôlent. Les études de résilience permettent ainsi d'interroger les capacités d'ajustement et d'adaptation naturelle des composantes physiques des territoires [Duvat et al., 2016] et d'apprentissage des sociétés, autrement dit de vérifier l'hypothèse selon laquelle les retours d'expérience post-événement jouent un rôle majeur dans le renforcement de la résilience des sociétés au fil du temps. En dépit de l'intérêt d'aborder la question de la gestion des risques naturels par la résilience, force est de constater d'une part, que les applications scientifiques concrètes du concept de résilience restent rares, et d'autre part, que ce concept est peu pris en compte dans les politiques publiques de gestion des risques et d'adaptation au changement climatique [Longépée, 2014].

Afin de contribuer à l'effort de recherche actuel, cet article s'attache à démontrer l'intérêt d'une approche de la résilience des territoires par la reconstruction de chaînes d'impacts d'événements météo-marins. Le concept de chaîne d'impacts a été développé par l'auteur principal dans un but opérationnel, et proposé au Ministère de l'Ecologie comme composante du référentiel national d'adaptation au changement climatique [ONERC, 2012]. Il désigne l'enchaînement et l'imbrication des impacts d'un événement naturel sur les différentes composantes d'un territoire (morphologie, ressources et écosystèmes, activités économiques, peuplement, etc.). Dans le cadre du projet de recherche RDT Réomers ${ }^{l}$ (2013-2016), ce concept a fait l'objet d'applications qui ont permis de vérifier non seulement sa pertinence pour évaluer la résilience des territoires, mais aussi son opérationnalité pour soutenir les politiques publiques de réduction des risques liés à la mer et d'adaptation au changement climatique. Cet article présente son application à des atolls des Tuamotu, en Polynésie française. L'atoll répond parfaitement à la notion de système complexe. En effet, la résilience de l'écosystème récifal (qui joue un rôle central) face aux perturbations climatiques et anthropiques détermine directement celle des sociétés humaines, en raison de la forte dépendance que cellesci entretiennent avec les composantes physiques du système. C'est finalement l'un des rares territoires occupés par des sociétés humaines dont l'habitabilité future sera conditionnée par les réponses de l'écosystème-support aux pressions qu'il subit [Duvat, 2015 ; Gattuso et al., 2015 ; Storlazzi et al., 2015 ; Karnauskas, 2016 ; Werner et al., 2017]. Le type de perturbation examiné ici est le cyclone tropical. Celui-ci constitue (i) le principal facteur naturel de déstabilisation de ces territoires [Larrue et Chiron, 2010 ; Laurent et Varney, 2014] (ii) un important facteur de risque futur, car le changement climatique engendre une augmentation de l'intensité des cyclones les plus intenses et une hausse (en voie d'accélération) de l'élévation du niveau de la mer. Cela devrait engendrer une hausse de la hauteur significative des vagues cycloniques, et du même coup, de leurs impacts destructeurs [Church et al., 2013 ; Hemer et al., 2013 ; Quataert et al., 2015].

Cet article présente la méthodologie adoptée et ses bénéfices pour la connaissance scientifique et pour les politiques publiques de réduction des risques liés à la mer et d'adaptation au changement climatique, en s'attachant à souligner: (1) l'intérêt de connaître la variabilité des impacts des événements météo-marins pour renforcer la résilience des territoires; (2) le rôle du processus d'apprentissage issu des retours d'expérience post-événement dans la réduction de la vulnérabilité des territoires à ces événements ; (3) les facteurs qui jouent le rôle de «barrière» dans la réduction des risques et l'adaptation au changement climatique ; (4) les processus complexes et intrinsèquement liés, physiques et humains, qui soutiennent la résilience des atolls, et qui requièrent la mise en œuvre de politiques d'adaptation spécifiques dans les territoires d'outre-mer français ; (5) le fait que les processus qui soutiennent la résilience d'un territoire, s'ils réduisent par certains leviers sa vulnérabilité, tendent aussi à faire émerger de nouvelles formes de vulnérabilité que les politiques publiques doivent anticiper et prendre en compte pour maximiser les bénéfices du processus d'apprentissage post-catastrophe [Duvat et al., 2016].

\section{DES ATOLLS RELATIVEMENT PEU EXPOSÉS AUX CYCLONES TROPICAUX}

Les trois atolls considérés, ceux de Rangiroa, Tikehau et Mataiva, sont situés entre $14^{\circ} 50^{\prime}$ et $15^{\circ} 20^{\prime} \mathrm{S}$, et entre $148^{\circ} 44^{\prime}$ et $147^{\circ} 10^{\prime} \mathrm{O}$, à l'extrémité nord-ouest de l'archipel des Tuamotu qui s'étire selon une

\footnotetext{
${ }^{1}$ RÉsilience des territoires d'Outre-MER aux risques météo-marins dans le contexte du changement global.
} 
orientation NO-SE sur près de $1500 \mathrm{~km}$ entre 14 et $23^{\circ} \mathrm{S}$. Ils appartiennent à la commune élargie de Rangiroa, qui inclut aussi l'île surélevée de Makatea, et présentent des caractéristiques contrastées. Si Rangiroa compte parmi les atolls les plus vastes $\left(1763 \mathrm{~km}^{2}\right.$ ) et les plus peuplés (2 $567 \mathrm{hab}$. en 2012, ISPF) des Tuamotu, Mataiva est l'un des plus petits $\left(47 \mathrm{~km}^{2}\right)$ et des moins peuplés, avec $272 \mathrm{hab}$. en 2012 (ISPF). Rangiroa est l'atoll le plus urbanisé de l'archipel, avec deux petits centres urbains situés aux extrémités ouest (Avatoru) et est (Tiputa) de sa zone aménagée, qui s'étire sur $13 \mathrm{~km}$ de la passe d'Avatoru à l'île de Tiputa incluse, alors que Tikehau et Mataiva sont des atolls ruraux, organisés autour d'un village qui compte quelques rues.

Le climat du nord-ouest des Tuamotu se caractérise par deux saisons [Andréfouët et al., 2012], l'été austral (nov.-mars), pendant lequel les alizés sont faibles et majoritairement de secteur E à NE, et l'hiver austral, pendant lequel ils sont plus soutenus et plus régulièrement issus du SE. Ces vents produisent une houle courte de secteur NE à SE, qui peut atteindre $4 \mathrm{~m}$ en saison fraîche. Les houles d'alizé peuvent être perturbées par des houles d'origine distante (tempérée ou polaire), en provenance du nord entre novembre et mars, et en provenance du sud entre avril et octobre. Ces houles à période longue peuvent dépasser 4 voire $5 \mathrm{~m}$, et avoir des impacts destructeurs sur les atolls (juillet 1996). Enfin, les cyclones surviennent pendant la saison chaude et à plus de $70 \%$ en situation El Niño, lorsque la «warm pool » du Pacifique ouest s'étend vers le Pacifique central. Sur la période 1970-2009, la Polynésie française a connu 44 épisodes cycloniques, qui ont principalement touché les archipels des Australes au sud et de la Société à l'ouest [Larrue et Chiron, 2010]. L'archipel des Tuamotu est moins affecté par les cyclones que les deux archipels précédents. Ceci étant, les atolls de l'ouest des Tuamotu, qui nous concernent ici, y sont les plus exposés : sur la période 19702009, ils ont connu en moyenne un cyclone tous les 8 à 10 ans. La saison cyclonique 1982-1983, influencée par un épisode El Niño exceptionnel, a vu la formation de 6 perturbations cycloniques à elle seule, dont 3 ont ravagé les atolls de Rangiroa, Tikehau et Mataiva (Tableau 1): Orama (passage à $100 \mathrm{~km}$ au SE du secteur habité de Rangiroa le 24 février 1983, à son paroxysme); Reva (passage à $50 \mathrm{~km}$ à l'ouest de Mataiva suivant une trajectoire NNE-SSO, dans la nuit du 8 au 9 mars 1983); et Veena (passage entre Mataiva et Tikehau le 11 avril 1983, suivant une trajectoire NNE-SSO) [Auzeneau et Darchen, 1983]. Ces cyclones n'ont fait qu'une dizaine de décès dans l'archipel des Tuamotu, en raison d'une vitesse de déplacement lente $(<15 \mathrm{~km} / \mathrm{h})$ qui a limité l'amplitude de la houle [Canavesio, 2015a], laissant le temps aux populations et aux autorités de mettre en place des mesures de protection [Dupon, 1987]. Lors de l'épisode El Niño exceptionnel suivant (1987-1998), la perturbation tropicale Veli, marquée par un déplacement très rapide $(>35 \mathrm{~km} / \mathrm{h})$, a ravagé Mataiva, lors de son passage à une centaine de kilomètres au SO de cet atoll le 31 janvier 1998. Depuis lors, ces trois atolls n'ont pas connu de dévastation par des cyclones.

Il s'agit donc ici d'étudier les impacts de cyclones et la résilience sur des territoires rarement touchés par ces phénomènes et sur lesquels les derniers événements remontent aux années 1980-1990.

\begin{tabular}{|c|c|c|c|c|}
\hline $\begin{array}{l}\text { Cyclone, passage sur } \\
\text { les Tuamotu et } \\
\text { catégorie (Saffir- } \\
\text { Simpson) }\end{array}$ & $\begin{array}{c}\text { Pression } \\
\text { barométrique } \\
\text { minimale }^{1} \\
(\mathrm{hPa})\end{array}$ & $\begin{array}{c}\text { Vitesse } \\
\text { moyenne des } \\
\text { vents }^{2}(\mathrm{~km} / \mathrm{h})\end{array}$ & $\begin{array}{c}\text { Hauteur } \\
\text { max. estimée de } \\
\text { la houle }\end{array}$ & $\begin{array}{c}\text { Principaux atolls touchés (sur les } \\
\text { atolls étudiés) et côtes les plus } \\
\text { affectés }{ }^{4}\end{array}$ \\
\hline $\begin{array}{l}\text { Orama } \\
\text { 17-24 février } 1983 \\
\text { Catégorie } 3\end{array}$ & 925 & 185 & $\begin{array}{l}9 \mathrm{~m} \text { côte sud } \\
\text { océanique de } \\
\text { Rangiroa et } 4,8 \mathrm{~m} \\
\text { côté lagon à } \\
\text { Tiputa }\end{array}$ & $\begin{array}{l}\text { Rangiroa (nord, est, sud-est, sud) } \\
\text { Tikehau (nord, est, sud-est, sud) }\end{array}$ \\
\hline $\begin{array}{l}\text { Reva } \\
\text { 8-14 Mars } 1983 \\
\text { Catégorie } 2\end{array}$ & 925 & 165 & $8-9 \mathrm{~m}$ & Mataiva (nord, ouest, sud-ouest) \\
\hline $\begin{array}{l}\text { Veena } \\
\text { 9-12 Avril } 1983 \\
\text { Catégorie } 3\end{array}$ & 925 & 185 & $10 \mathrm{~m}$ & $\begin{array}{l}\text { Tikehau (nord, est) } \\
\text { Rangiroa (nord, est) } \\
\text { Mataiva (nord, est, sud-est) }\end{array}$ \\
\hline $\begin{array}{l}\text { Veli } \\
30 \text { janvier-3 février } \\
1998 \\
\text { Catégorie } 1\end{array}$ & 980 & 120 & $7-8 \mathrm{~m}$ & Mataiva (nord, ouest, sud) \\
\hline
\end{tabular}

Tableau 1. Caractéristiques des cyclones étudiés 
Sources : ${ }^{1}$ Bureau of Meteorology, Australian Government $;{ }^{2}$ Estimations du JTWC $;{ }^{3}$ Modélisations de Damlamian et Kruger (2013), de Lecacheux et al. (2013) et de MetOcéan Solutions Ltd ; ${ }^{4}$ Auzeneau et Darchen (1983), Larrue and Chiron (2010), et entretiens réalisés auprès de la population et des autorités publiques.

\section{MÉTHODE DE RECONSTRUCTION DE CHAINES D'IMPACTS D'EVENEMENTS PASSES}

Dans des territoires comme les atolls des Tuamotu, la pratique désormais répandue en France des RETEX est difficilement mobilisable faute d'événements récents. La reconstruction de chaînes d'impacts d'événements cycloniques passés constitue alors une alternative intéressante en ce qu'elle permet de reconstituer les impacts d'événements « de référence » ainsi que les enchaînements d'impacts qui ont produit la catastrophe. Ce travail de reconstruction s'appuie sur quatre démarches complémentaires :

(1) Une recherche bibliographique et documentaire visant à renseigner quels cyclones ont affecté les territoires étudiés (date, durée de vie, trajectoire, intensité, houle associée), lesquelles de leurs manifestations (vent, houle, pluie, etc.) ont eu des impacts, et quels ont été précisément ces impacts.

(2) La réalisation d'entretiens semi-directifs auprès de personnes ayant vécu ces événements, afin d'en préciser les impacts à l'échelle de l'atoll et des îles habitées. Le choix des interrogés s'est appuyé sur trois critères : (i) le vécu direct de l'événement, (ii) une répartition la plus régulière possible des interrogés entre les îles et façades littorales affectées, (iii) la constitution d'un panel d'interrogés représentatif de l'ensemble des enjeux du territoire (politiques, religieux, économiques, etc.). L'identification des personnes à interroger a été facilitée par la dimension restreinte des communautés considérées. La fiabilité des témoignages a été évaluée sur la base des informations fournies pendant l'entretien, puis par leur confrontation avec celles fournies par les autres interrogés. Seuls les entretiens considérés comme fiables ont été conservés et traités. Les entretiens ont duré une heure et demie en moyenne, et ont porté sur les 8 types d'impacts recensés dans la littérature scientifique : (1) sur les îles (érosion, submersion, etc.) ; (2) sur le récif corallien; (3) sur les ressources et les écosystèmes ; (4) sur les activités de subsistance ; (5) sur les activités économiques ; (6) sur les infrastructures et les services fondamentaux ; (7) démographiques et sanitaires ; (8) sur le bâti [Nurse et al., 2014 ; Duvat et al., 2016]. Pour chaque type d'impact, les questions ont porté sur sa nature, son intensité (ex. : hauteur d'eau), les processus qui l'ont généré, son caractère négatif et/ou positif selon l'interrogé, la zone d'impact (levée au GPS pour les impacts proches du lieu de résidence, et représentée par l'interrogé sur une carte pour les impacts éloignés), sa durée, et les modalités de retour à la normale. Des photographies et documents illustrant les impacts ont pu être collectés auprès de certains interrogés, complétant utilement les témoignages. Le nombre d'entretiens par événement, compris entre 4 et 13 , correspond au nombre de personnes ayant vécu l'événement qui habitent encore sur place et ont pu en retranscrire les impacts avec fiabilité.

(3) L'analyse comparée des photographies aériennes prises avant et après les cyclones, datées des 20/10/1981 et 27/03/1984 pour Rangiroa et de Tikehau, et des 4/02/1976 et 27/03/1984 pour Mataiva, qui a permis de mesurer l'évolution de la position du trait de côte due à ces cyclones, et de localiser les zones d'accumulation sédimentaire associées à ces événements [Duvat et Pillet, 2017 ; Duvat et al., à paraître].

(4) La retranscription des impacts de ces cyclones sous la forme de chaînes d'impacts événementielles (une chaîne d'impacts par événement et par territoire), puis génériques (une chaîne d'impacts par cyclone ou par type de cyclone). Si la connaissance des impacts s'est appuyée sur les trois démarches précédentes, le « chaînage » des impacts a été reconstitué sur la base de l'expertise scientifique des auteurs.

\section{RÉSULTATS ET DISCUSSION}

\section{IV.1 IMPACTS DES CYCLONES DE 1983 À RANGIROA, MATAIVA ET TIKEHAU}

Les cyclones de $1983^{2}$ (de catégorie 2 pour Reva et 3 pour Veena et Orama), ont été très destructeurs : ils ont intégralement ravagé les atolls qu'ils ont touchés (Figure 1), et certains de leurs impacts ont perduré plus d'une décennie.

\footnotetext{
${ }^{2}$ Lorsque deux cyclones se sont succédé en un à deux mois, comme cela été le cas d'Orama (23-24/02) et de Veena (10-11/04) à Rangiroa, et de Reva (6-14/03) et Veena (10-11/04) à Mataiva, les habitants ont des difficultés à distinguer leurs impacts respectifs, le second cyclone ayant en général aggravé les impacts du premier. Dans ce cas, les impacts décrits sont des impacts cumulés.
} 
Inondation-submersion et salinisation de la lentille d'eau souterraine et des sols : Les inondations ont été causées principalement par le débordement des marécages sous l'effet des précipitations et de l'élévation temporaire du niveau de la mer, et localement aggravées, dans les zones urbanisées, par l'imperméabilisation des sols et le remblaiement des marécages, comme à Avatoru (Rangiroa) où la zone remblayée est restée sous $1 \mathrm{~m}$ d'eau pendant deux semaines. Les côtes exposées aux vagues cycloniques ont été submergées : dans la partie centrale de la zone aménagée de Rangiroa, les îles ont été successivement submergées par le sud sur la moitié de leur largeur pendant le passage d'Orama, puis par le nord, sur le tiers à la moitié de leur largeur lors du passage de Veena. De même, dans la zone habitée de Mataiva, la succession des cyclones Reva et Veena à un mois d'intervalle a engendré la submersion de la côte océanique par Reva, puis celle de la côte lagonaire par Veena. À cela, il faut ajouter la réactivation d'anciens hoa ${ }^{3}$ aujourd'hui comblés et incorporés aux îles, qui s'est traduite par la formation de couloirs de submersion reliant la côte océanique à la côte lagonaire. Là où deux cyclones se sont succédé, la combinaison de ces processus fait que peu d'espaces intérieurs ont échappé à la submersion. Celle-ci a affecté la qualité de la lentille d'eau souterraine et des sols, en engendrant leur salinisation. Sur l'île de Tuherahera à Tikehau, la salinisation de la lentille a empêché son exploitation pendant 2 à 10 ans suivant les secteurs. De la même manière, la salinisation des sols les a rendus inexploitables sur des périodes de 2 mois à Rangiroa après Orama et Veena, de 6 mois à Mataiva après Reva et Veena, et de 10 ans à Tikehau après le passage de Veena. En parallèle, l'eau de pluie stockée par les particuliers dans des citernes en ciment a été polluée par les intrusions d'eau marine et les décombres. Là où les deux sources d'approvisionnement (citerne et lentille souterraine) ont été affectées, la situation a été critique: suite aux cyclones de 1983, l'atoll de Mataiva a été dépendant de l'extérieur pour son approvisionnement en eau pendant un an, le temps de reconstruire les systèmes de collecte et de stockage.

Impacts morpho-sédimentaires : Les vagues cycloniques de 1983 ont engendré d'importantes modifications morpho-sédimentaires [Duvat et al., à paraître] : (i) un recul ou une avancée du trait de côte dépassant $30 \mathrm{~m}$ par endroits ; (ii) des transferts de sédiments des plages vers l'intérieur des terres, sous la forme de nappes d'épandage engendrant un exhaussement des îles de $0,50 \mathrm{~m}$ à $1 \mathrm{~m}$ par endroits ; (iii) le transfert de débris et blocs de corail des pentes externes du récif vers le platier conglomératique, les côtes et l'intérieur des terres, les plus gros blocs dépassant $1 \mathrm{~m}$ de diamètre; (iv) la contraction, la fragmentation ou la migration vers le lagon de certains petits îlots $(<1 \mathrm{ha})$, en particulier dans le sud de Tikehau. Ces impacts morpho-sédimentaires ont affecté la qualité et l'exploitabilité des sols (recouvrement par des débris et blocs).

Dégradation des écosystèmes et des ressources : Dans les trois atolls, les colonies coralliennes ont été détruites à $60-100 \%$ sur les 20-30 premiers mètres des pentes externes du récif, et sur la face ouest de Tikehau, jusqu'à 80 à $100 \mathrm{~m}$ de profondeur sous l'effet d'avalanches de débris [Harmelin-Vivien et Laboute, 1986]. Le suivi de la résilience des pentes externes par les biologistes marins a révélé un retour à la situation pré-cyclonique en 10 ans [Duvat et al., à paraître]. Dans le lagon de Tikehau, des patates de corail de $2 \mathrm{~m}$ de diamètre pesant $1 \mathrm{t}$ ont été arrachées et retournées lors du passage de Veena. La végétation indigène du littoral a été en grande partie arrachée par le vent et déracinée par les vagues, mais elle montrait déjà une bonne reprise un an après les cyclones. L'analyse des photographies aériennes confirme non seulement sa régénération, mais aussi dans certains cas (nord de Mataiva), sa croissance plus rapide dans les secteurs dévastés par les cyclones que sur le reste du linéaire côtier entre 1984 et 1994 [Duvat et al., à paraître].

Impacts sur l'économie de subsistance et sur l'économie marchande : Le fait que les ressources des atolls (noix de coco et poisson, principalement) soient peu diversifiées, et qu'elles soient à la fois consommées (en dépit de l'importance des produits importés dans l'alimentation) et commercialisées, démultiplie les impacts socioéconomiques des cyclones, car ceux-ci détruisent les ressources alimentaires et privent en même temps la population des revenus monétaires nécessaires pour acheter des produits importés. Après les cyclones de 1983, on note d'abord une forte diminution des prises de pêche, en raison de la perturbation du milieu et de la faune - sur une durée de 6 mois à 1 an dans le lagon à Rangiroa - et de la destruction des parcs à poissons (sauf à Mataiva) et parfois aussi des aménagements et équipements de pêche. À Tikehau par exemple, le village des pêcheurs situé au nord de la passe a été intégralement détruit, quais compris. Cela, ajouté à la perturbation du milieu lagonaire, a engendré une forte réduction des prises de pêche pendant 5 ans, ce qui a affecté l'alimentation de la population. En parallèle, la destruction quasi-totale des cocoteraies par le vent (à $95 \%$ à Tikehau après le passage de Veena, par ex.), a interrompu la production de coprah pendant près de 3 ans, à Tikehau comme à Rangiroa. Cependant, suite à la replantation des cocoteraies qui a bénéficié d'un important soutien du gouvernement [Dupon, 1987], la production de coprah

\footnotetext{
${ }^{3}$ Chenal inter-1̂le (profondeur maximale de 1 à $3 \mathrm{~m}$ en général).
} 
était plus élevée trois ans après les cyclones que l'année qui les a précédés : à Tikehau, elle était de 600 t/an en 1986 contre 400 t/an avant Veena; de la même manière, à Mataiva, la production de 1989 dépassait celle de 1982. En détruisant les cocoteraies, les cyclones ont imposé leur régénération qui avait été jusque-là négligée. S'ils ont donc annihilé la production pendant plusieurs années, ils ont été bénéfiques à plus long terme.

Dégâts sur les infrastructures et les réseaux : L'accès à l'électricité a été interrompu pendant 3 jours à Rangiroa après Orama et Veena, et près d'un mois à Mataiva après Reva et Veena. Les infrastructures de transport sont parfois restées inutilisables pendant une longue période, comme dans le cas de l'aérodrome de Mataiva qui n'a repris son activité qu'au bout d'un an, et des routes à Tikehau dont le nettoyage manuel des débris qui les encombraient a pris 6 mois. À Rangiroa, il a fallu reconstruire 4 des 7 ponts qui traversent les hoa dans la zone aménagée ainsi que les quais, ce qui a pris 6 mois. Après chaque cyclone, le trafic aérien a par ailleurs été interrompu pendant une période de 48 à $72 \mathrm{~h}$, en raison de l'inondation de la piste par le débordement du marécage et des incursions d'eau marine (à Tikehau, en particulier), ou de son recouvrement par des débris et des blocs de corail (à Rangiroa).

Destruction quasi-totale du bâti, y compris des bâtiments à fonction de refuge : Les cyclones de 1983 ont détruit la quasi-totalité des habitations à Tikehau $(90 \%$ des toits arrachés par le vent, $95 \%$ de destruction) et à Mataiva, où il a fallu deux ans pour reconstruire. À Tiputa (Rangiroa), $80 \%$ des habitations ont été ravagées par le vent et par les vagues, qui ont détruit celles situées en première et deuxième lignes côté lagon, ce qui s'est aussi produit à Avatoru. Certains bâtiments servant de refuge (églises, mairies ou écoles), ont également été endommagés. La destruction de la majorité des habitations, peu résistantes car constituées de bois et de panneaux de contreplaqué, avec des toits de tôle mal fixés au corps de l'habitation, a rendu nécessaire une aide massive du gouvernement pour la reconstruction. Il s'en est suivi une amélioration de la résistance de l'habitat, qui a significativement réduit la vulnérabilité de la population des atolls face aux cyclones. Mais bien qu'ils aient démontré la forte vulnérabilité physique des habitants des Tuamotu, les événements de 1983 n'ont engendré dans ces atolls ni la construction systématique d'abris anticycloniques [Canavesio, 2015b]), ni l'introduction massive des habitations modulaires anticycloniques, dispositifs pourtant introduits dans d'autres atolls (ex. : Anaa [Canavesio, 2015a]) par l'Agence Territoriale de la Reconstruction [Dupon, 1987].

Déplacements de population : Les cyclones de 1983 n'ont pas fait de morts dans les trois atolls considérés ici, car leurs populations ont pu se réfugier dans des bâtiments en dur (église ou temple, mairie). Quelques blessés ont été évacués vers Tahiti. Sur le plan démographique, ces cyclones ont surtout engendré des déplacements de population, au sein des atolls, des zones agricoles dévastées vers la zone urbanisée ou le village, et des atolls affectés vers Tahiti, pour des familles qui ont perdu leurs moyens de production. Ces déplacements de population vers Tahiti ont concerné une vingtaine de personnes à Mataiva, soit $10 \%$ des habitants, et ils ont dans la plupart des cas été temporaires, suivis d'un retour au bout de quelques années au maximum. Les aides accordées par le gouvernement pour relancer la production de coprah dans les Tuamotu ont permis la reprise économique rapide de cette filière, ce qui a favorisé le retour des habitants dans leur atoll, dans un laps de temps compris entre 3 et 10 ans selon les cas. 


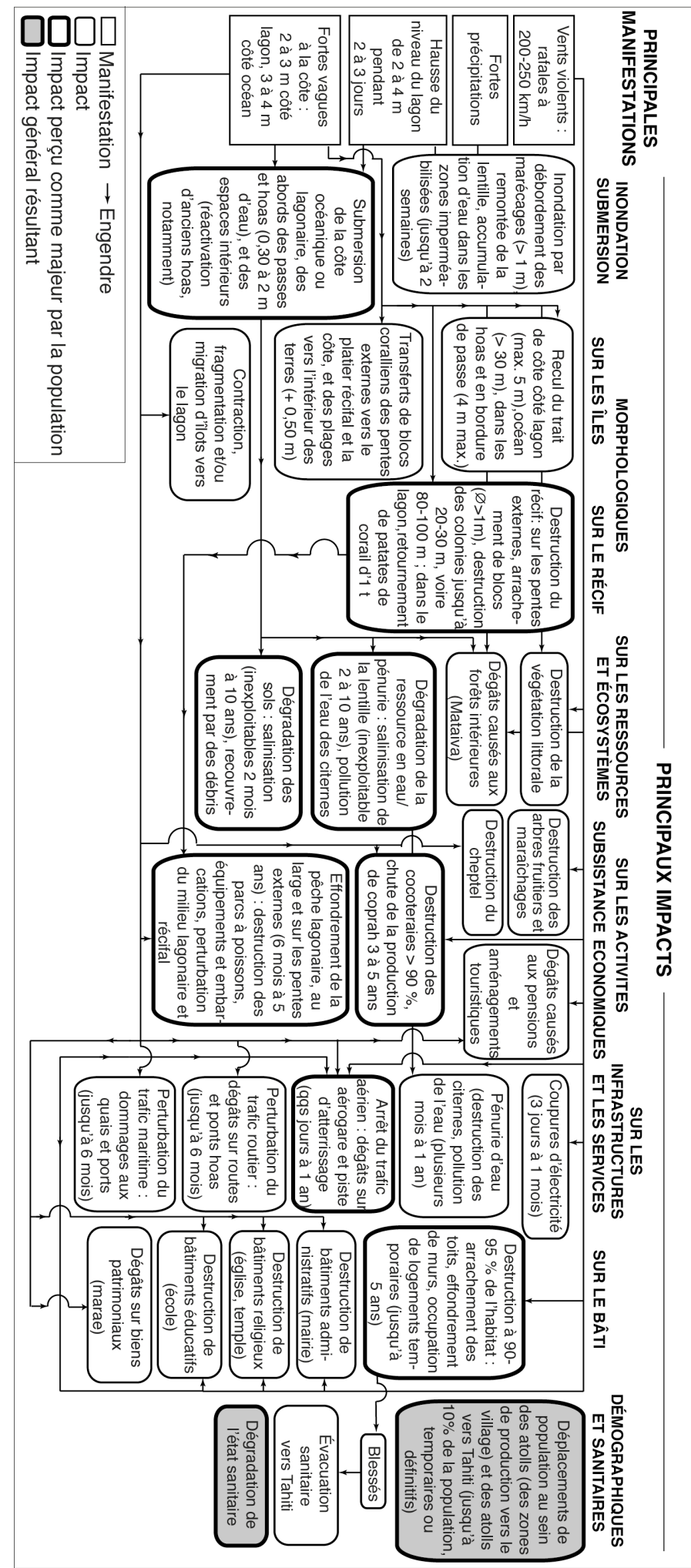

Figure 1. Chaîne d'impacts des cyclones de 1983 à Rangiroa, Tikehau et Mataiva, Polynésie française.

\section{IV.2 IMPACTS DU CYCLONE VELI (FÉVRIER 1998) SUR L’ATOLL DE MATAIVA}

Début février 1998, l'atoll de Mataiva a été atteint par le nord-ouest par la houle générée par la perturbation tropicale Veli (catégorie 1), synchronisée avec une houle distante significative en provenance du Pacifique Nord. Cette houle a été annoncée par le service météorologique de la Polynésie française, mais fortement sous-estimée : les vagues qui ont déferlé sur le récif dans le nord-ouest de l'atoll, où se situe le village, ont atteint 7 à $8 \mathrm{~m}$ de hauteur au lieu des 3-4 m annoncés. 
À la différence de ce qui s'était produit en 1983, les houles de Veli ont eu une zone d'impact restreinte à l'échelle de l'atoll, circonscrite à sa face ouest (Figure 2A). Ni la production de coprah, ni les prises de pêche de l'atoll n'ont par conséquent été affectées. Dans le secteur du village, cette houle cyclonique a eu de très forts impacts (Figure 2B) : elle a traversé les îles de la côte océanique au lagon, en répandant sur son passage d'importantes quantités de sable, blocs coralliens et débris (cocotiers, portions de la piste d'atterrissage, murs d'habitations...), dont le charriage a contribué à endommager le bâti et les infrastructures. Il a fallu deux ans pour réparer les dégâts causés à la piste d'atterrissage et à l'aérogare. Les deux-tiers des bâtiments du village ont été directement endommagés par les vagues. Ceux situés à l'est, protégés par les précédents, ont été envahis par le sable et l'eau sur une hauteur atteignant $2 \mathrm{~m}$ dans les zones les plus basses situées du côté du lagon (Figure 2B). Sur la côte ouest de Mataiva, la puissance de cette houle pourtant issue d'une perturbation tropicale peu intense a été plus forte que celle des houles des cyclones de 1983, comme l'attestent le déplacement et la fracturation de blocs cyclopéens, le déchaussement des fondations des bâtiments et le creusement de vastes dépressions ici et là dans le village. Certains débris arrachés dans la zone du village ont été retrouvés jusqu'à l'atoll de Tikehau.

L'étendue de la submersion explique par ailleurs que la lentille d'eau souterraine et les sols aient été inexploitables pendant 5 ans, et que certains habitants aient fait venir de la terre de Tahiti pour pouvoir replanter arbres fruitiers et légumes. L'ensemble de la production maraîchère et fruitière, ainsi que le petit cheptel de poulets et chèvres ont été anéantis, et ces productions n'ont depuis lors jamais retrouvé leur niveau d'avant 1998. Comme en 1983, l'eau des citernes est devenue impropre à la consommation, ce qui a rendu nécessaire le largage d'eau potable par avion pendant plusieurs mois afin de subvenir aux besoins de la population.

Comme les précédents, cet événement a mis en évidence la forte dépendance des atolls vis-à-vis de Tahiti en situation de crise et de post-crise. En raison de ses impacts très dévastateurs, la houle cyclonique de 1998 a été à l'origine de changements importants qui ont eu pour effet de réduire significativement la vulnérabilité de la population aux événements météo-marins. Elle a d'abord engendré la construction d'un bâtiment anticyclonique permettant de mettre à l'abri la population en cas d'événement. Parce que ce bâtiment est utilisé comme école, son statut d'abri est discuté aujourd'hui, mais il n'en demeure pas moins qu'il est conforme aux normes anticycloniques et constitue un abri pour l'ensemble de la population. Par ailleurs, parce qu'il a détruit un grand nombre d'habitations et est survenu après la mise sur le marché des premières habitations anticycloniques, le cyclone de 1998 a favorisé leur introduction à Mataiva : $15 \%$ des habitations de cet atoll sont des fare MTR (habitation anticyclonique) contre moins de $7 \%$ à Rangiroa. Le troisième bénéfice de cet événement a été la constitution d'un talus de protection du village avec les matériaux naturels et les débris d'origine anthropique produits et charriés par le cyclone : bien que ce talus ne constitue pas dans son état actuel une protection suffisante, il traduit physiquement la forte exposition de la partie sud du village aux houles cycloniques (position dans l'axe de la passe), renforçant la conscience du risque des habitants. Enfin, le dernier impact bénéfique de long terme de cet événement a été, comme pour les cyclones de 1983, de stimuler la production de coprah, ce qui a accru les revenus de la population. 




Figure 2. Cartographie des impacts du cyclone Veli (30-01/03-02-1998) sur l'atoll de Mataiva, Polynésie française. A. Zone d'impact et impacts à l'échelle de l'atoll. B. Cartographie des impacts dans le village.

\section{IV.3 DISCUSSION ET CONCLUSION : RENFORCER LES MESURES DE PRÉVENTION DES RISQUES, UNE STRATÉGIE «SANS REGRETS »}

Comme on l'a vu, il existe différents types de cyclones destructeurs (1983 vs. 1998) générant des impacts variés, non seulement par leur nature, mais aussi par leur empreinte spatiale. Les cyclones intenses ne sont ni les seuls cyclones dévastateurs, ni forcément les plus destructeurs. Des événements de faible intensité (catégorie 1), mais à déplacement rapide comme Veli, produisent également des houles particulièrement destructrices. L'absence de signes annonciateurs (vent et pluie), la sous-estimation possible et l'amplification 
rapide de la houle rendent finalement la population plus vulnérable à ce type d'événement qu'à un cyclone puissant, mais dont le déplacement est plus lent. Les entretiens réalisés à Mataiva le confirment : « contre le vent, on peut se mettre à l'abri, renforcer nos toitures et nos fenêtres, attacher nos maisons au sol, mettre nos voitures et engins de construction dans des abris, etc., mais contre la mer, on ne peut pas faire grandchose. » (entretien du 13/02/2015 auprès d'Edgar Tetua, maire de Mataiva en 1998). Par ailleurs, la reconstruction des cartographies d'impacts des cyclones passés montre que les côtes océaniques comme lagonaires peuvent être submergées sur une largeur importante, atteignant localement $200 \mathrm{~m}$ et donc excédant largement les $10 \mathrm{~m}$ côté lagon et $30 \mathrm{~m}$ côté océan qui ont été retenus comme "zone d'aléa fort à majeur » pour l'élaboration des Plans de prévention des risques (PPR) dans les atolls de Polynésie.

Dans ce contexte, la priorité doit être de renforcer les mesures de prévention, en privilégiant : (1) La mise en sécurité de la population dans des abris anticycloniques. Or, si Tikehau et Mataiva ont fini par être équipés d'abris anticycloniques, la construction des trois abris nécessaires à la mise en sécurité de la population de Rangiroa n'a toujours pas été engagée, pour des raisons principalement financières et foncières. Or, elle s'impose au vu de la difficulté qu'il y aurait à évacuer les 2567 habitants de cet atoll en cas d'événement. (2) La réduction de la vulnérabilité structurelle des bâtiments collectifs et des habitations par leur surélévation, en appliquant la norme existante de 1,50 m. Si jusqu'aux années 1940 à 1960, les habitations étaient construites sur des dalles de 0,80 à $1,50 \mathrm{~m}$ de hauteur, donc surélevées et situées au-dessus des niveaux d'eau les plus fréquemment atteints, cette pratique s'est perdue suite au développement de l'habitat moderne en dur construit à même le sol et à la perte de la mémoire du risque. Elle pourrait être réactivée par la généralisation des maisons modulaires anticycloniques, dont l'acquisition par les particuliers reste limitée alors qu'elle est fortement subventionnée, et donc facilitée, par le gouvernement. Ces habitations ne représentent à ce jour que 7 et $9 \%$ respectivement des habitations de Rangiroa et de Tikehau, contre $15 \%$ de celles de Mataiva [Ranché et Magnan, 2016]. L'absence de généralisation de ces habitations s'explique d'une part, par la faible conscience du risque et le relatif fatalisme des habitants des atolls, et d'autre part, par une difficulté d'application réelle de la réglementation relative à l'urbanisation et à l'aménagement du territoire, qui tient à différents facteurs : l'éloignement des atolls par rapport à Tahiti, qui réduit l'application de la loi, les conflits qui opposent les acteurs locaux aux représentants du Territoire (ces derniers incarnant l'Etat) et plus globalement le refus d'un système " français », donc exogène, dans un territoire dans lequel les problèmes posés par l'introduction du cadastre dans les années 1960 ne sont toujours pas réglés. Comme les habitants des atolls, les élus locaux considèrent que la réglementation relative aux PPR, qui définit des bandes côtières inconstructibles de $10 \mathrm{~m}$ et de $30 \mathrm{~m}$ côtés lagon et océan respectivement, n'est pas adaptée au régime foncier d'indivision, et pas acceptable sans compensation financière des contraintes qu'elle impose (perte de terrain "occupable »). De fait, la mise en place des PPR est aujourd'hui gelée. Or une mesure telle que la surélévation de l'habitat aurait de nombreux bénéfices : permettre aux îles de s'exhausser sous l'effet des transferts de sédiments dus aux tempêtes, ce qui réduirait le risque de submersion; réduire les dégâts causés aux bâtiments par leur mitraillage par les débris charriés par les vagues de tempête; et réduire la construction de digues et de murs de protection, qui se généralise alors que ces ouvrages sont peu efficaces et accroissent la vulnérabilité de la population en lui donnant un faux sentiment de sécurité. (3) L'édification de talus de protection des villages implantés dans l'axe des passes et exposés aux houles cycloniques, comme celui de Mataiva, qui reste mal protégé par le talus édifié à partir des débris de 1998. (4) La constitution de stocks d'eau et de nourriture, qui seraient bienvenus dans l'attente de l'aide extérieure en cas d'événement. Si les cyclones de 1983 ont utilement soutenu l'acquisition par les particuliers de collecteurs d'eau pluviale plus adaptés que les anciens réservoirs en ciment, ils ont cependant engendré l'abandon de la plupart des cultures de fruits et légumes et de l'élevage, ce qui a renforcé le rôle des importations dans l'alimentation, donc des revenus monétaires, dans la vie des habitants des atolls, et a engendré des problèmes de santé publique (diabète, cholestérol).

Dans le contexte du changement climatique, privilégier la prévention en s'attachant à appliquer des mesures prenant en compte à la fois les processus naturels et les intérêts de la population, telles que la généralisation d'abris et d'un modèle d'habitations anticycloniques, sont « sans regrets » puisqu'elles répondent aux enjeux actuels (résilience) comme futurs (anticipation). Les verrous que constituent le manque de moyens financiers mobilisables, le régime foncier d'indivision et la faiblesse de la loi en général, ne pourront être levés que si une véritable stratégie collaborative, de l'échelon local à l'échelon national (Territoire et France) et régional (Pays polynésiens ou Petits Etats insulaires), est élaborée. L'impossibilité pour la Polynésie, en tant que territoire français, d'accéder au Fonds internationaux pour l'adaptation, rend d'autant plus crucial le fait d'engager une réflexion concertée sur la réduction des risques et l'adaptation au changement climatique. 


\section{REMERCIEMENTS}

Les auteurs remercient le MEDDE-MEEM, qui a financé le projet Réomers-RDT (13-MRES-RDT-1-CVS022), et l'ANR pour son soutien aux projets CapAdapt (ANR-11-JSH1-0004) et Storisk (ANR-15-CE030003), ainsi que Marien Ranché, Fanny Rubia et Adrien Prenveille pour leur contribution à la collecte des données, et les acteurs locaux et habitants qui ont mis à leur disposition informations et documents.

\section{REFERENCES}

Andrefouët S. et al. (2012). - Island shadow effects and the wave climate of the Western Tuamotu Archipelago (French Polynesia) inferred from altimetry and numerical model data. Marine Pollution Bulletin, 65: 415-424.

Auzeneau S., Darchen J. (1983). - Autour de la saison 1982-1983 des perturbations tropicales en Polynésie Française. Météorologie maritime, 120 : 14-30.

Canavesio R. (2015a). - Estimer les houles cycloniques à partir d'observations météorologiques limitées : exemple de la submersion d'Anaa en 1906 aux Tuamotu (Polynésie française). VertigO, 14(3), URL: http://vertigo.revues.org/15384

Canavesio R. (2015b). Politiques publiques de gestion des submersions aux Tuamotu : L'exemple de l'aménagement des abris anticycloniques. Bulletin de la Société des Etudes Océaniennes, n 334, pp. 24-39

Church J.A. et al. (2013). - Sea level change. In: Stocker, T.F., Qin, D., Plattner, G.K., Tignor, M., Allen, S.K., Boschung, J., Nauels, A., Xia, Y., Bex, V., Midgley, P.M. (Eds.) Climate Change: The Physical Science Basis. Contribution of Working Group 1 to the Fifth Assessment Report of the Intergovernmental Panel on Climate Change. Cambridge University Press, pp. 1137-1216.

Dupon J.-F. (1987). - Les atolls et le risque cyclonique. Le cas des Tuamotu. Cahier des Sciences Humaines, 23(S-4) : $567-599$.

Duvat V. (2015). - Changement climatique et risques côtiers dans les îles tropicales. Les Annales de Géographie, 705 : $541-566$.

Duvat V., Magnan A., Etienne S., Salmon C., Pignon-Mussaud C. (2016). - Assessing the impacts of and resilience to Tropical Cyclone Bejisa, Reunion Island (Indian Ocean). Natural Hazards, 83: 601-640.

Duvat V., Pillet, V. (2017). - Shoreline changes in reef islands of the Central Pacific: Takapoto Atoll, Northern Tuamotu, French Polynesia. Geomorphology, 282: 96-118.

Duvat V., Salvat B., Salmon C. (à paraître). - Drivers of shoreline change in atoll reef islands of the Tuamotu Archipelago, French Polynesia. Global and Planetary Change.

Gattuso, J.-P., Magnan, A., Billé, R., Cheung, W.W.L., Howes, E.L., Joos, F., Allemand, D., Bopp, L., Cooley, S.R., Eakin, C.M., Hoegh-Guldberg, O., Kelly, R.P., Pörtner, H.-O., Rogers, A.D., Baxter, J.M., Laffoley, D., Osborn, D., Rankovic, A., Rochette, J., Sumaila, U.R., Treyer, S., Turley, C. (2015). - Contrasting futures for ocean and society from different anthropogenic $\mathrm{CO}_{2}$ emissions scenarios. Science, 349: aac4722.

Harmelin-Vivien M.L., Laboute P. (1986). - Catastrophic impact of hurricanes on atoll outer slopes in the Tuamotu (French Polynesia). Coral Reefs, 5: 55-62.

Hemer, M.A., Fan, Y., Mori, N., Semedo, A., Wang, X.L. (2013). - Projected changes in wave climate from a multi-model ensemble. Nature Climate Change, 3 : 471-476.

Institut de la Statistique de Polynésie Française (ISPF), 2012. Recensement de population.

Karnauskas, B.K., Donnelly, J.P., Anchukaitis, K.J. (2016). - Future freshwater stress for island populations. Nature Climate Change Letters.

Larrue S., Chiron T. (2010). - Les îles de Polynésie française face à l'aléa cyclonique. VertigO, 10(3), URL : http://vertigo.revues.org/10558

Laurent, V., Varney, P. (2014). - Historique des cyclones de Polynésie française de 1831 à 2010. Météo France, Tahiti, 172 p.

Longépée E. (2014). - La résilience des systèmes socio-écologiques des états atolliens dans le contexte du changement climatique : le cas des Kiribati (Pacifique Central). Thèse de géographie, Université de la Rochelle.

Nurse L., et al. (2014). - Small Islands, in V.R. Barros et al. (eds.), Climate Change 2014: Impacts, Adaptation and Vulnerability Contribution of Working Group II in the Fifth Assessment Report of the Intergovernmental Panel on Climate Change, Cambridge University Press, pp.1613-1655.

ONERC (2012). - Les outre-mer face au défi du changement climatique. Rapport au Premier Ministre et au Parlement, Paris, La Documentation Française.

Quataert, E., Storlazzi, C., an Rooijen, A., Cheriton, O., van Dongeren, A. (2015). - The influence of coral reefs and climate change on wave-driven flooding of tropical coastlines. Geophysical Research Letters, 42: 6407-6415.

Ranché M., Magnan A.K. (2016). - Caractérisation de l'habitat des atolls de Rangiroa et Tikehau, Polynésie française. Rapport de projets (CapAdapt et Réomers), Iddri, Paris, 26 p.

Storlazzi, C.D., Elias, E. P. L., Berkowitz, P., 2015. Many Atolls May Be Uninhabitable Within Decades Due to Climate Change. Nature Scientific Reports, 5, 14546.

Walker B., Holling C.S., Carpenter S.R., Kinzig A. (2004). - Resilience, Adaptability and Transformability in Social-ecological Systems. Ecology and Society, 9(2), 9 p.

Werner, A.D., Sharp, H.K., Galvis, S.C., Post, V.E.A., Sinclair, P. (2017). - Hydrogeology and management of freshwater lenses on atoll islands: review of current knowledge and research needs. Journal of Hydrology. 